\title{
El Catálogo General del Patrimonio Histórico de Andalucía
}

\author{
Juan Manuel Becerra García \\ Jefe del Servicio de Protección de \\ Patrimonio Histórico
}

Fuensanta Plata García

Jefa del Dpto. de Régimen

General

María José Fitz Canca

Documentalista

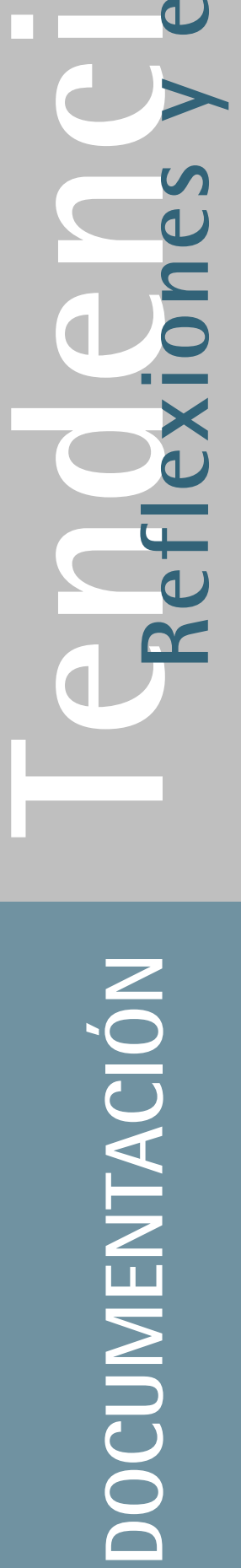
artículos 149.1 y 149.2 de la Constitución". ña, Galicia y País Vasco. coge que las Comunidades Autónomas con una acción autonómica específica, caso de la Comunidad Autónoma de Andalucía, según el Estatuto de Autonomía y la Ley I/199| de Patrimonio Histórico de Andalucía y sus Reglamentos de Desarrollo, gozan de competencias concurrentes con el Estado especialmente en materia de inventario y declaración de bien de interés cultural, con lo que se regula la potestad de la Comunidad Autónoma Andaluza en esta materia.

La Ley |/|99| de 3 de Julio de Patrimonio Histórico de Andalucía presenta entre otras medidas la "creación del Catálogo General de Patrimonio Histórico Andaluz como instrumento para la salvaguarda de los bienes en él inscritos, la consulta y la divulgación del mismo...". Para individualizar las medidas protectoras la Ley recoge "la posibilidad de que la catalogación vaya acompañada del establecimiento de instrucciones particulares de modo que se eviten los problemas que la aplicación de normas genéricas puede plantear... Estas instrucciones permiten además acotar el grado de discrecionalidad de la Administración en su actuación posterior, ya que el otorgamiento o denegación de las autorizaciones exigidas por la Ley deberá, lógicamente, realizarse teniendo en cuenta las instrucciones citadas". bre traspaso de funciones y servicios del Estado a la Comunidad en materia de Cultura se traspasan todas las funciones sobre Patrimonio Histórico en los términos recogidos en el Anexo I, apartado B, punto Ia, que son las siguientes "todas las funciones sobre Patrimonio Histórico, Artístico, Monumental, Arquitectónico, Arqueológico, Paleontológico, Etnológico y sobre el tesoro bibliográfico y documental a salvo de lo que disponen los

Sin embargo, la Ley 16/1985 de 25 de Junio, de Patrimonio Histórico Español, reserva para el Estado en exclusiva una de las principales competencias sobre Patrimonio Histórico, cual es la de incluir en Inventario General o declarar de interés cultural los bienes culturales, por lo que fue recurrida ante el Tribunal Constitucional por las Comunidades Autónomas de Catalu-

La Sentencia del Tribunal Constitucional núm. 17/1991, de 31 de Enero de 1991, a este respecto re-
Igualmente en materia de protección la Ley crea dos nuevas figuras sin precedente en la legislación histórica, como son la Zona de Servidumbre Arqueológica y el Lugar de Interés Etnológico. En la aplicación de la primera de ellas, se exige que el planeamiento urbanístico tenga en cuenta la posible existencia de restos arqueológicos y se instituye una notificación previa que permita a la administración cultural realizar los estudios previos que resulten necesarios para evitar la posible suspensión de un proyecto ante la aparición de hallazgo en mitad de su ejecución. La segunda figura se aplica a los lugares, edificios o instalaciones que merezcan ser objeto de una atención especial por constituir en sí mismos o albergar en su seno elementos constitutivos del patrimonio etnográfico de Andalucía.

El Catálogo General del Patrimonio Histórico Andaluz queda regulado en la Ley de Patrimonio Histórico Andaluz a través de los artículos 6-12, ambos inclusive. En

La nueva Sección Tendencias: reflexiones y experiencias se concibe como un espacio de encuentro para la presentación de experiencias, propuestas metodológicas, proyectos de investigación y de innovación tecnológica, estudios y reflexiones que enriquezcan el panorama de la información y la documentación del patrimonio histórico. 
el artículo 6 se define y se atribuye la competencia para su formación y conservación a la Consejería de Cultura, que igualmente debe hacerse cargo de la redacción y de la custodia de la documentación correspondiente a los bienes culturales que constituyen el Patrimonio Histórico Andaluz. Así mismo se establece que el Catálogo General será de pública consulta, quedando la documentación administrativa sometida a las normas establecidas para el Patrimonio Documental.

Las inscripciones en el Catálogo pueden tener carácter genérico, cuando se pretenda únicamente identificar un bien como parte integrante de dicho Patrimonio, o con carácter específico cuando se quieran alicar las normas generales y particulares especialmente previstas en la Ley para esta clase de inscripciones.

Igualmente también forman parte del Catálogo General, según establece el artículo I 3 de la Ley andaluza, los bienes declarados de interés cultural (B.I.C) con arreglo a la Ley 16/1985, de 25 de Junio.

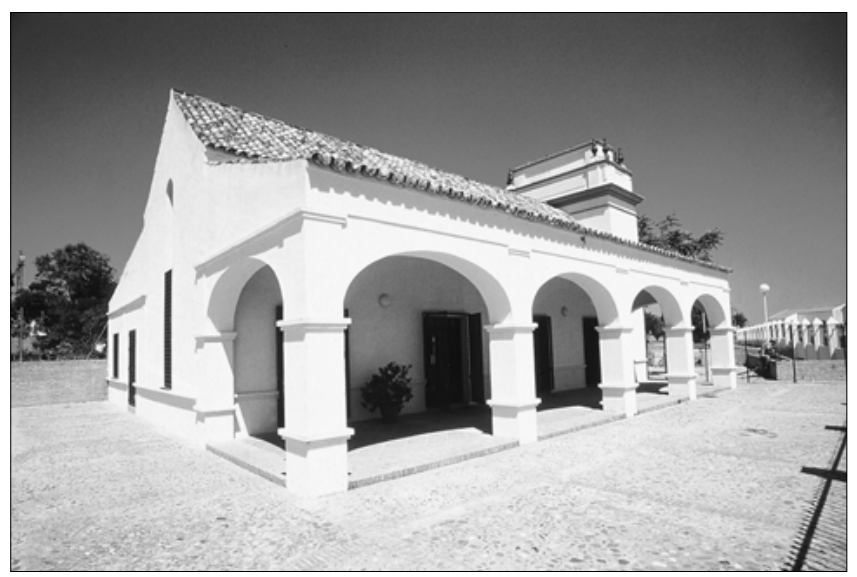

Hacienda Sacra Familia ca o genérica. Los aproximadamente 250 restantes están incluidos en Inventario General de Bienes Muebles. Sin protección legal, dado que sus expedientes sólo están en fase de solicitud sin haberse producido aún la incoación, se encuentran aproximadamente 650 bienes para su declaración como B.I.C. y otros 375 para su inscripción, bien específica bien genérica.

El Reglamento de Protección y Fomento, en su artículo 3, punto 4, sistematiza el conjunto total de bienes estableciendo una clasificación general por secciones atendiendo a las figuras de protección, que es la siguiente:

a) Bienes con inscripción genérica.

b) Bienes con inscripción específica.

c) Bienes declarados de interés cultural.

Atendiendo a la tipología de los bienes, la clasificación señalada por el Reglamento en el punto 2 del mismo artículo 3, establece las categorías que se enumeran a continuación:

a) Patrimonio Inmueble.

b) Patrimonio Mueble.

c) Actividades de Interés Etnológico.

d) Patrimonio Documental.

e) Patrimonio Bibliográfico.

Para el Patrimonio Inmueble, en caso de inscripción específica, en el Anexo I del Reglamento de Protección y Fomento del Patrimonio Histórico de Andalucía, se recogen seis tipologías:
I. Monumento.
2. Conjunto Histórico.
3. Zona Arqueológica.
4. Jardín Histórico
5. Sitio Histórico.
6. Lugares de Interés Etnológico.

A estas tipologías hay que añadir las señaladas por el Reglamento de Desarrollo I I I/I986, de 10 de Enero, de la Ley de Patrimonio Histórico Español, para los B.I.C. incluidos en la categoría de Patrimonio Inmueble, que son las siguientes:
I. Monumento.
2. Jardín Histórico.
3. Conjunto Histórico.
4. Sitio Histórico.
5. Zona Arqueológica.

Para el Patrimonio Mueble, en caso de inscripción específica, en el Anexo I del Reglamento de Protección y Fomento del Patrimonio Histórico de Andalucía, se recogen 27 tipologías, entre las cuales constan entre otras: pintura, escultura, retablística, orfebrería, textiles, vidrieras, cerámica, mobiliario, instrumentos musicales, maquinaria y objetos para uso técnico y científico, objetos e instrumentos de interés etnográfico, etc. aproximadamente 2.300 bienes, de los cuales unos I.900 gozan de categoría de B.I.C., de ellos 400 todavía en tramitación; un segundo grupo está compuesto por 150 bienes, aproximadamente, con inscripción específ--
El Patrimonio Mueble con declaración de B.I.C. o inclusión en Inventario General, según el Reglamento de 
Desarrollo II I/1986, de 10 de Enero, de la Ley de Patrimonio Histórico Español, Anexos I.c, I.d y I.e, se clasifica del siguiente modo: bienes muebles, materiales arqueológicos, numismática y epigrafía; y materiales etnográficos.

La categoría de Actividades de Interés Etnológico se contempla de forma exclusiva en el Reglamento de Protección y Fomento del Patrimonio Histórico Andaluz, para las figuras de inscripción genérica o específica, no estableciéndose ninguna clasificación tipológica.

El Patrimonio Documental, en caso de inscripción específica, se clasifica en el Anexo I del Reglamento de Protección y Fomento del Patrimonio Histórico de Andalucía, en dos tipologías: Documentos y Archivos; y el Reglamento de Desarrollo I I I/1986, de I0 de Enero, de la Ley de Patrimonio Histórico Español, clasifica los B.I.C. e Inventario General en Manuscritos, Impresos y Colecciones (Anexos I.h, I.i y I.j).

El Anexo I del Reglamento de Protección y Fomento del Patrimonio Histórico igualmente clasifica en dos tipologías, en caso de inscripción específica, el Patrimonio Bibliográfico: Libros y Bibliotecas. El Reglamento de Desarrollo I I I/I986, de 10 de Enero, de la Ley de Patrimonio Histórico Español, lo clasifica en los Anexos I.h, I.i y I.j, como Manuscritos, Impresos y Colecciones, respectivamente, tanto para la declaración de B.I.C. como para la inclusión en Inventario General.

Actualmente de todas las categorías que contempla la legislación vigente, la más desarrollada en el Catálogo General corresponde a la categoría de Patrimonio Inmueble, correspondiendo el índice más elevado a las declaraciones de B.I.C., en sus tipologías de Monumentos, Conjuntos Históricos y Zonas Arqueológicas, con claro predominio del primer grupo. Por su importancia numérica, le sigue a esta categoría la de Patrimonio Mueble, no obstante el gran desfase que las separa. El resto de las categorías -Actividades de Interés Etnológico, Patrimonio Documental y Patrimonio Bibliográfico- no han sido objeto de inscripciones en el Catálogo, bajo ninguna de las figuras recogidas en ambas legislaciones reguladoras.

\section{La documentación que configura los expedientes del Catálogo General}

El Catálogo General del Patrimonio Histórico Andaluz por ser un instrumento administrativo y científico, tal como lo define la Ley de Patrimonio Histórico de Andalucía, está compuesto por unidades documentales, los expedientes, configurados por documentos de diversa índole, siendo fundamental en su composición la documentación administrativa y la documentación técnica.

Respecto a la primera, regulada por la Ley 30/92, de 26 de Noviembre, de Régimen Jurídico de las Administraciones Públicas y del Procedimiento Administrativo Común, está integrada por la documentación generada en las distintas fases de la tramitación: incoación, notificaciones, información pública, audien-

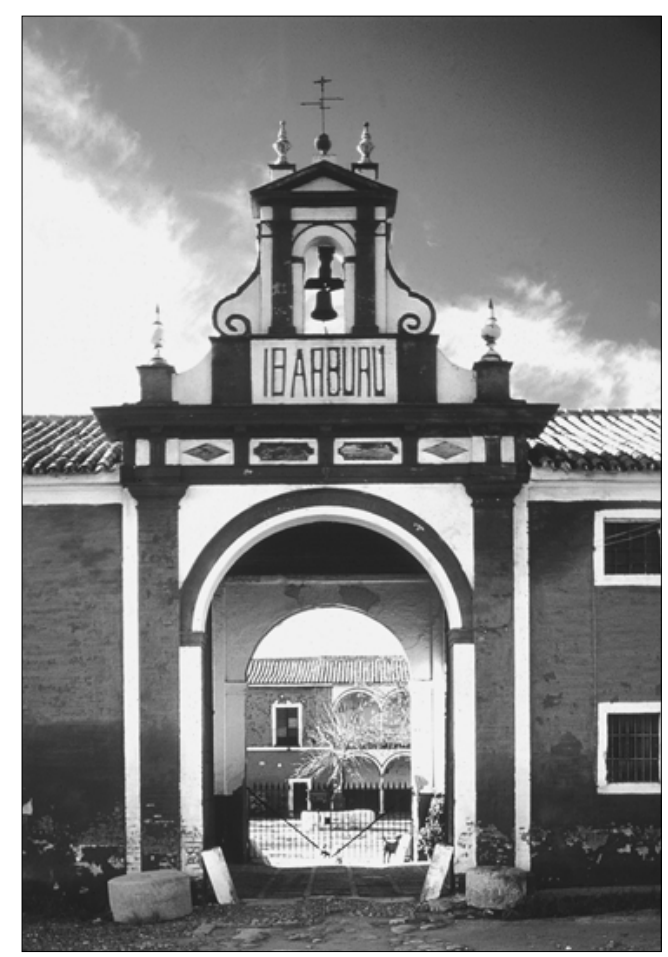

cia a interesados y ayuntamientos, informes de institución consultiva, resolución y sus correspondientes publicaciones en BOJA y BOE. Esta compleja secuencia queda simplificada en los casos en los que se trata de inscripciones o declaraciones de bienes muebles, patrimonio documental y bibliográfico. Su importancia radica en que su correcta ejecución asegura la viabilidad del régimen de protección idóneo para el bien de que se trate, facilitando la tutela jurídico-administrativa del Patrimonio Histórico Andaluz.

La documentación técnica viene regulada en los Anexos de los Reglamentos de Desarrollo tanto de la ley estatal como de la ley andaluza. Esta parte del expediente, frente a la anterior, es más singularizada, ya que persigue la identificación específica del bien objeto de inscripción, debe ser realizada con metodología científica y constituye la principal fuente de consulta del Catálogo respecto a los bienes que lo componen, dotándolo de este modo de su carácter científico y contribuyendo a la divulgación y conocimiento del Patrimonio Histórico Andaluz.

El expediente técnico de cada bien recoge información relativa a:

- Localización

- Denominación.

- Código de identificación.

- Tipología o clasificación (sólo en caso de bienes objeto de inscripción específica).

- Justificación de la inscripción del bien en el Catálogo General del Patrimonio Histórico.

- Descripción del bien.

- Delimitación del inmueble objeto de inscripción (sólo en caso de inmuebles).

- Bienes muebles vinculados al inmueble.

- Datos histórico-artísticos. 


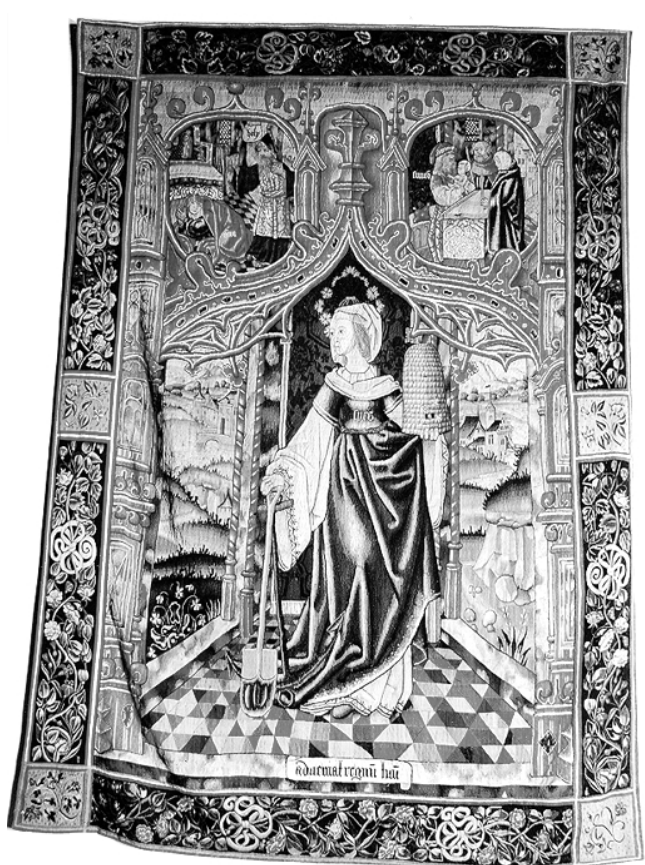

Tapiz Gótico La Esperanza. Anónimo. Pizarra (Málaga)

\section{SISTEMA DE CATALOGACIÓN DEL P.H. EN ANDALUCÍA}

\begin{tabular}{|c|c|c|c|}
\hline \multirow{27}{*}{$\begin{array}{l}\text { CATÁLOGO } \\
\text { GENERAL } \\
\text { DEL PATRIMONIO } \\
\text { HISTÓRICO } \\
\text { ANDALUZ }\end{array}$} & CATEGORÍA & SECCIÓN & TIPOLOGÍA \\
\hline & \multirow{13}{*}{$\begin{array}{l}\text { PATRIMONIO } \\
\text { MUEBLE }\end{array}$} & \multicolumn{2}{|l|}{ INSCRIPCIÓN GENÉRICA } \\
\hline & & \multirow[t]{7}{*}{ INSCRIPCIÓN ESPECÍFICA } & MONUMENTO \\
\hline & & & JARDÍN HISTÓRICO \\
\hline & & & CONJUNTO HISTÓRICO \\
\hline & & & SITIO HISTÓRICO \\
\hline & & & ZONA ARQUEOLÓGICA \\
\hline & & & LUGAR DE INTERÉS \\
\hline & & & ETNOLÓGICO \\
\hline & & \multirow{5}{*}{$\begin{array}{l}\text { DECLARADOS DE INTERÉS } \\
\text { CULTURAL }\end{array}$} & MONUMENTO \\
\hline & & & JARDÍN HISTÓRICO \\
\hline & & & CONJUNTO HISTÓRICO \\
\hline & & & SITIO HISTÓRICO \\
\hline & & & ZONA ARQUEOLÓGICA \\
\hline & \multirow{3}{*}{$\begin{array}{l}\text { PATRIMONIO } \\
\text { INMUEBLE }\end{array}$} & \multicolumn{2}{|l|}{ INSCRIPCIÓN GENÉRICA } \\
\hline & & INSCRIPCIÓN ESPECÍFICA & 27 Tipologías \\
\hline & & \multicolumn{2}{|c|}{ DECLARADOS DE INTERÉS CULTURAL } \\
\hline & \multirow{2}{*}{$\begin{array}{l}\text { ACTIVIDADES DE } \\
\text { INTERÉS ETNOLÓGICO }\end{array}$} & \multicolumn{2}{|l|}{ INSCRIPCIÓN GENÉRICA } \\
\hline & & \multicolumn{2}{|l|}{ INSCRIPCIÓN ESPECÍFICA } \\
\hline & \multirow{4}{*}{$\begin{array}{l}\text { PATRIMONIO } \\
\text { DOCUMENTAL }\end{array}$} & \multicolumn{2}{|l|}{ INSCRIPCIÓN GENÉRICA } \\
\hline & & \multirow{2}{*}{ INSCRIPCIÓN ESPECÍFICA } & DOCUMENTO \\
\hline & & & ARCHIVO \\
\hline & & \multicolumn{2}{|c|}{ DECLARADOS DE INTERÉS CULTURAL } \\
\hline & \multirow{4}{*}{$\begin{array}{l}\text { PATRIMONIO } \\
\text { BIBLIOGRÁFICO }\end{array}$} & \multicolumn{2}{|l|}{ INSCRIPCIÓN GENÉRICA } \\
\hline & & \multirow{2}{*}{ INSCRIPCIÓN ESPECÍFICA } & LIBRO \\
\hline & & & BIBLIOTECA \\
\hline & & \multicolumn{2}{|c|}{ DECLARADOS DE INTERÉS CULTURAL } \\
\hline \multicolumn{4}{|c|}{ ZONA DE SERVIDUMBRE ARQUEOLÓGICA } \\
\hline \multicolumn{4}{|c|}{ INVENTARIO GENERAL DE BIENES MUEBLES } \\
\hline
\end{tabular}

INVENTARIO GENERAL DE BIENES MUEBLES

- Estado de conservación.

- Descripción y análisis del planeamiento urbanístico y territorial (sólo en caso de bienes inmuebles objeto de inscripción específica).

- Fuentes documentales y bibliográficas.

- Situación jurídica.

- Observaciones.

- Datos administrativos.

- Documentos gráficos.

- Instrucciones particulares (sólo en caso de bienes objeto de inscripción específica).

Éstas últimas son objeto de desarrollo en el artículo 16 del Reglamento de Protección y Fomento del Patrimonio Histórico, y tienen como finalidad concretar para cada bien objeto de inscripción específica y su entorno, la forma en que deben materializarse para los mismos, las obligaciones generales previstas en la Ley |/I99| de Patrimonio Histórico y en el mismo Reglamento para los propietarios o poseedores de dichos bienes.

El contenido de las mismas es el siguiente:

- Condicionantes previos a la intervención en el bien catalogado o en los inmuebles de su entorno.

- Intervenciones, actividades, elementos y materiales que pueden ser aceptables y aquellos otros expresamente prohibidos.

- Tipos de obras o actuaciones sobre el bien catalogado o su entorno en las que no será necesaria la presentación del Proyecto de Conservación.

- Medidas a adoptar para preservar el bien de acciones contaminantes y de variaciones atmosféricas, térmicas o higrométricas.

- Técnicas de análisis que resulten adecuadas.

- Determinación de las reproducciones o análisis susceptibles de llevar aparejado algún tipo de riesgo para el bien y que, en consecuencia, quedan sujetos al régimen de autorización tanto de la Consejería de Cultura como del titular del bien.

- Definición de aquellos inmuebles incluidos en Conjuntos Históricos inscritos en el Catálogo General del Patrimonio Histórico Andaluz cuya demolición podrá autorizarse sin necesidad de declaración de ruina.

- Régimen de investigación aplicable al bien catalogado y a los inmuebles incluidos en el entorno.

- Señalamiento de los inmuebles sitos en Conjuntos Históricos o en el entorno de bienes catalogados a cuyas transmisiones pueda aplicarse el derecho de tanteo y retracto.

- Cualquier otra determinación consecuencia de los deberes de conservación, mantenimiento y custodia que se considere necesario matizar o concretar.

El cuadro siguiente ofrece una visión sintética del Sistema de Catalogación del Patrimonio Histórico en Andalucía que recoge las propuestas formuladas por la Ley 16/1985 de 25 de Junio, de Patrimonio Histórico Español, por la Ley |/1999| de 3 de Julio, de Patrimonio Histórico de Andalucía y por sus respectivos reglamentos de desarrollo. 